\title{
Inferring hot-star-wind acceleration from Line Profile Variability
}

\author{
L. Dessart ${ }^{1}$ and S. P. Owocki ${ }^{2}$ \\ 1 Max-Planck-Institut für Astrophysik, Karl-Schwarzschild-Str. 1, 85748 Garching bei München, Germany \\ e-mail: luc@mpa-garching.mpg.de \\ 2 Bartol Research Institute of the University of Delaware, Newark, DE 19716, USA \\ e-mail: owocki@bartol.udel.edu
}

Received 14 June 2004 / Accepted 6 November 2004

\begin{abstract}
The migration of profile sub-peaks identified in time-monitored optical emission lines of Wolf-Rayet (WR) star spectra provides a direct diagnostic of the dynamics of their stellar winds via a measured $\Delta v_{\mathrm{LOS}} / \Delta t$, a line-of-sight velocity change per unit time. Inferring the associated wind acceleration scale from such an apparent acceleration then relies on the adopted intrinsic velocity of the wind material at the origin of this variable pattern. Such a characterization of the Line Emission Region (LER) is in principle subject to inaccuracies arising from line optical depth effects and turbulence broadening. In this paper, we develop tools to quantify such effects and then apply these to reanalyze the LER properties of time-monitored WR stars. We find that most program lines can be fitted well with a pure optically thin formation mechanism, that the observed line-broadening is dominated by the finite velocity extent of the LER, and that the level of turbulence inferred through Line Profile Variability $(l p v)$ has only a minor broadening effect in the overall profile. Our new estimates of LER velocity centroids are systematically shifted outwards closer to terminal velocity compared to previous determinations, now suggesting WR-wind acceleration length scales $\beta R_{*}$ of the order of 10-20 $R_{\odot}$, a factor of a few smaller than previously inferred. Based on radiation-hydrodynamics simulations of the line-driven-instability mechanism, we compute synthetic lpv for CIII5696 A for WR 111. The results match well the measured observed migration of $20-30 \mathrm{~m} \mathrm{~s}^{-2}$, equivalent to $\beta R_{*} \sim 20 R_{\odot}$. However, our model stellar radius of $19 R_{\odot}$, typical of an O-type supergiant, is a factor 2-10 larger than generally expected for WR core radii. Such small radii leave inferred acceleration scales to be more extended than expected from dynamical models of line driving, which typically match a "beta" velocity law $v(r)=v_{\infty}\left(1-R_{*} / r\right)^{\beta}$, with $\beta \approx 1-2$; but the severity of the discrepancy is substantially reduced compared to previous analyses. We conclude with a discussion of how using lines formed deeper in the wind would provide a stronger constraint on the key wind dynamics in the peak acceleration region, while also potentially providing a diagnostic on the radial variation of wind clumping, an issue that remains crucial for reliable determination of O-star mass loss rates.
\end{abstract}

Key words. line: formation - radiative transfer - stars: atmospheres - stars: early-type - stars: mass-loss

\section{Introduction}

The hot, massive, luminous stars of spectral type $\mathrm{O}, \mathrm{B}$, and WR are understood to have strong stellar winds driven by the linescattering of the star's continuum radiation (see, e.g., Puls \& Kudritzki 2000). For OB stars, dynamical models based on the line-driving formalism of Castor et al. (1975, hereafter CAK) and its modern extensions (Pauldrach et al. 1986; Friend \& Abbott 1986) yield predictions for the mass loss rate $\dot{M}$ and terminal speed $v_{\infty}$ that are generally in quite good agreement with observationally inferred values (Puls et al. 1996; Crowther et al. 2002). Indeed, for these relatively optically thin winds, the formation of line profiles throughout the flow acceleration region yields a constraint on the full wind velocity law, commonly characterized through the phenomenological "beta-law" form $v(r)=v_{\infty}\left(1-R_{*} / r\right)^{\beta}$, where $R_{*}$ is the (hydrostatic) surface value of the radius $r$, and the velocity exponent $\beta$ is inferred from line profile fits. Careful time-monitoring of optical emission lines (e.g. He II 4686; Eversberg et al. 1998) show that these winds are not strictly steady, but likely consist of quite extensive small-scale structure. This leads to an intrinsic, low-level, Line Profile Variability $(l p v)$, characterized by moving subpeaks that provide an even more direct diagnostic of the wind acceleration. For OB stars, both the single exposure line profiles and such lpv subpeak monitoring suggest a velocity-law exponent $\beta \approx 1-1.2$, which is roughly consistent with the value $\beta \approx 0.8-1$ predicted from dynamical models of line-driving.

Over evolutionary timescales, the cumulative depletion of the hydrogen-rich stellar envelope of such OB stars is understood to lead to the Wolf-Rayet (WR) stage, characterized by reduced or no hydrogen, with spectra dominated by broad emission lines of He and CNO processed material. For such WR stars, the very large mass loss rates inferred from the strength of these wind-broadened emission lines are difficult to reconcile with a detailed, dynamical model based on the 
paradigm of radiative driving via line-scattering. Because the higher density makes the inner wind optically thick even in the electron scattering continuum, there are now no direct diagnostics of the initial acceleration from the hydrostatic stellar core, making it difficult to constrain dynamical models. Moreover, the overall shape of the emission profile turns out to be relatively insensitive to even the acceleration scale in the outer part of the wind (see Sects. 3.1-3.2).

For such WR winds, the monitoring of moving subpeaks from $l p v$ thus provides a crucial diagnostic of the outer wind acceleration (Robert 1992). The most extensive previous analysis of such $l p v$ is that of Lépine \& Moffat (1999, hereafter LM). By combining the measured subpeak acceleration with an estimate of the characteristic velocity and range of the Line Emission Region (LER), they inferred wind acceleration scales $\beta R_{*} \approx 20-80 R_{\odot}$. If applied to typical OB supergiant radii $R_{*} \approx$ 20-45 $R_{\odot}$ (Crowther et al. 2002; Hillier et al. 2003), such acceleration scales imply a velocity index $(\beta \approx 1-2)$, which is nearly compatible with the value predicted by wind models. But both evolutionary (Langer et al. 1994; Schaerer \& Maeder 1992) and spectroscopic (Hillier \& Miller 1999) models of WR stars suggest a much smaller hydrostatic core radius, $R_{*} \approx 2-5 R_{\odot}$, for which the LM results imply a very large ratio between acceleration scale and core radius, formally $\beta \approx 4-40$, with a typical value $\beta \approx 10$. Over an associated factor 10 range in radius the radiative flux declines by a factor of $1 / 100$, making it difficult to understand how such extended acceleration scales could be maintained by a radiative driving mechanism.

This difficulty motivates our present reexamination of the basic inference of wind acceleration scales from $l p v$, with particular emphasis on the assumed velocity and width of the LER. We first provide a simple overview (Sect. 2) of how a measured acceleration can be related to a characteristic acceleration scale through an associated velocity range. We next (Sect. 3) review the sensitivity of the overall shape of emission profiles to the wind and LER parameters, showing thereby that the observed emission line broadenings are consistent with relatively large and extended velocities for the LER, without need to invoke large turbulent speeds. We then (Sect. 4) show that such larger and more extended LER velocities imply substantially lower acceleration scales $\left(\beta R_{*} \approx 10-20 R_{\odot}\right)$, which are now much closer to being compatible with dynamical models that account for the multi-line scattering expected in dense, WR winds. In Sect. 5 we examine how well $l p v$ and associated subpeak accelerations can be reproduced through dynamical simulations of the intrinsic instability of line-driving. Finally, Sect. 6 summarizes our results and provides an outlook for future work.

\section{Dynamics of wind acceleration}

\subsection{Acceleration length scale}

To gain a basic understanding of the factors influencing an inferred wind acceleration scale, consider first the simple case of constant acceleration $a$ between a starting velocity $v_{1}$ and final velocity $v_{\infty}$. By simple dynamics, the associated length is

$s_{1}=\frac{v_{\infty}^{2}-v_{1}^{2}}{2 a}$

For application to a stellar wind, let us scale the starting velocity by the terminal value, $w_{1} \equiv v_{1} / v_{\infty}$, and the length by a solar radius,

$\frac{s_{1}}{R_{\odot}}=\frac{1-w_{1}^{2}}{2 \tilde{a}}$,

where $\tilde{a} \equiv a R_{\odot} / v_{\infty}^{2}$ represents a convenient scaling for the acceleration. Applying now the acceleration magnitudes inferred by $\mathrm{LM}$ from WR $l p v, \tilde{a} \approx 0.001-0.005$, we immediately derive associated length scales

$\frac{s_{1}}{R_{\odot}}=100-500\left(1-w_{1}^{2}\right)$.

If we now associate the quantity $w_{1}$ defined above with the characteristic fractional velocity of the inner edge of the LER, we see that the typical LER speeds assumed by LM, $w_{1} \approx 0.6$, imply very large acceleration scales, $s_{1} / R_{\odot} \approx 60-300$. By contrast, if we instead take $w_{1} \approx 0.9$ (see Sect. 3.2, Fig. 4), we find $s_{1} / R_{\odot} \approx 20-100$.

An alternative way to characterize the overall wind acceleration is to make use of the phenomenological beta-velocity law

$w(r) \equiv v(r) / v_{\infty}=\left(1-R_{*} / r\right)^{\beta}$,

for which the scaled acceleration vs. velocity becomes

$\tilde{a}=R_{\odot} w w^{\prime}=\frac{\beta R_{\odot}}{R_{*}}\left(1-w^{1 / \beta}\right)^{2} w^{2-1 / \beta}$.

However, since $w<1$, note that for moderately large $\beta$,

$w^{1 / \beta}=\mathrm{e}^{(1 / \beta) \ln w} \approx 1+(1 / \beta) \ln w$,

which implies

$\tilde{a} \approx \frac{R_{\odot}}{\beta R_{*}}(w \ln w)^{2}$.

This very useful property, which was first derived by Lépine (1998), implies that a plot of acceleration vs. speed just scales homologously with the parameter $\beta R_{*}$ (see Fig. 8 below). For an assumed scaled velocity of the LER, $w_{l}$, this now gives a scaled acceleration length,

$\frac{\beta R_{*}}{R_{\odot}} \approx \frac{\left(w_{1} \ln w_{1}\right)^{2}}{\tilde{a}}$

Comparison with Eq. (2) show that the beta-law characterization of the acceleration scale differs from that for constant acceleration by the replacement of numerator $\left(1-w_{1}^{2}\right)$ with $\left(w_{1} \ln w_{1}\right)^{2}$. Applying now the LM values for the velocity of the LER, $w_{1} \approx 0.6$, we find $\beta R_{*} / R_{\odot} \approx 20-90$. By contrast, taking $w_{1} \approx 0.9$ gives $\beta R_{*} / R_{\odot} \approx 2-9$.

Note that these are much smaller than the simple, local scale given by Eq. (2). The reason for the difference is illustrated in Fig. 1, which plots the distance to reach to half-way 


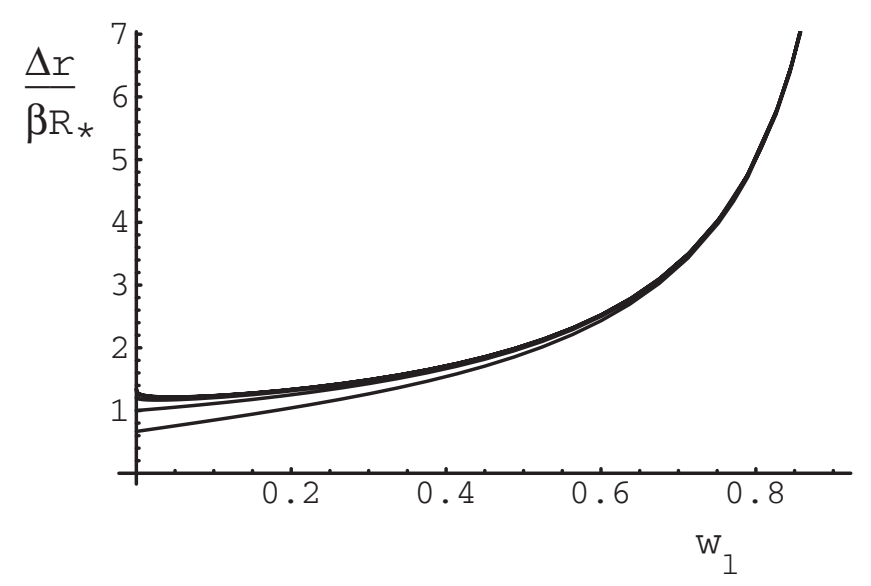

Fig. 1. The radial distance to reach from a given scaled velocity $w_{l}$ to half-way to the terminal speed, $\left(1+w_{1}\right) / 2$, scaled by $\beta R_{*}$, and plotted vs. $w_{1}$ for various velocity indices $\beta=1 / 2$ (lowermost curve) and $\beta=1,2,4,6,8$, and 10 (overplotted curves).

to terminal speed as a function of initial speed, scaled by the characteristic scale $\beta R_{*}$, and overplotted for various values of the velocity index $\beta$. The order unity values for small initial velocities shows that $\beta R_{*}$ is indeed a characteristic acceleration scale for the inner regions of a beta velocity law; but at large velocities, the local acceleration scales are actually much larger.

\subsection{Simple overview of radiative driving}

For a steady-state, spherical wind with velocity $v(r)$ as a function of radius $r$, the net flow acceleration $a=v(\mathrm{~d} v / \mathrm{d} r)$ results from the outward driving against the inward pull of gravity,

$v \frac{\mathrm{d} v}{\mathrm{~d} r}=g_{\mathrm{rad}}-\frac{G M}{r^{2}}$

where $G$ and $M$ are the gravitation constant and stellar mass. For WR and other very luminous stars, the general belief is that the outward driving is from stellar radiation interacting with both line and continuum opacity of wind material. For a star of luminosity $L$, the radiative flux is $F=L / 4 \pi r^{2}$, implying that overall the radiative acceleration tends to have the same inverse-radius-squared decline as gravity. Since gravity moreover sets the overall acceleration scale, it is convenient to define the gravitationally scaled accelerations $u^{\prime} \equiv r^{2} v(\mathrm{~d} v / \mathrm{d} r) / G M$ and $\Gamma_{\text {rad }} \equiv r^{2} g_{\text {rad }} / G M$.

For driving associated with continuum opacity $\kappa_{\mathrm{c}}, g_{\mathrm{rad}}=$ $\kappa_{\mathrm{c}} F / c$, and so

$\Gamma_{\mathrm{c}}=\frac{\kappa_{\mathrm{c}} L}{4 \pi G M c}$.

In the common case that the continuum is dominated by electron scattering opacity $\kappa_{\mathrm{e}}\left(\approx 0.34 \mathrm{~cm}^{2} / \mathrm{g}\right)$, this is a constant that just reduces the effective gravity by a factor $1-\Gamma$, with $\Gamma$ the classical Eddington parameter.

By contrast, for line opacity, the Doppler-shift deshadowing of optically thick lines gives line driving a dependence on the flow acceleration, parameterized within the CAK formalism as

$\Gamma_{1}=f C u^{\prime \alpha}$,

where $0<\alpha<1$ is the CAK power-index, $f$ is a complex function of velocity, radius, and acceleration that accounts for the finite size of the stellar disk (see CAK Eq. (50)), and $C$ is a constant set by line-opacity, luminosity, and mass loss rate.

With these scalings, the equation of motion (9) can thus be written as

$u^{\prime}=f C u^{\prime \alpha}-(1-\Gamma)$.

For the idealized case of a point-source star, $f=1$, this equation has no explicit radial dependence, implying that each term is just a fixed constant. A trivial radial integration of the scaled acceleration $u^{\prime}$ from a static lower boundary stellar radius $R_{*}$ thus yields the velocity law

$v(r)=v_{\infty}\left(1-R_{*} / r\right)^{1 / 2}$,

where the terminal speed $v_{\infty}=v_{\text {esc }} \sqrt{u^{\prime}}$ is proportional to the effective surface escape speed, $v_{\text {esc }} \equiv \sqrt{2 G M(1-\Gamma) / R_{*}}$. The critical CAK solution with the maximal mass loss rate further requires

$1=\frac{\partial \Gamma_{1}}{\partial u^{\prime}}$

which when combined with Eq. (12) implies a critical acceleration $u_{\mathrm{c}}^{\prime}=C_{\mathrm{c}}=\alpha /(1-\alpha)$, yielding $v_{\infty}=v_{\mathrm{esc}} \sqrt{\alpha /(1-\alpha)}$.

For more complete models that take account of the finite stellar disk (Friend \& Abbott 1986; Pauldrach et al. 1986), the reduction of the line-force near the star (where $f<1$ ) lowers both the initial acceleration and the mass loss rate. But as $f$ increases outward - with $f \rightarrow 1$ at large radii, where the stellar radiation indeed approaches the radial streaming from a point-source - the lower mass loss rate allows a much stronger acceleration $\left(u^{\prime} \approx 6-9\right.$ !), thus yielding a faster wind terminal speed, $v_{\infty} / v_{\text {esc }}=2.5-3$. The resulting velocity law is no longer analytic, but can still be well fit by a general "beta-law" form (4), with now $\beta \approx 0.8-1$ (Pauldrach et al. 1986).

For WR stars, the importance of line overlap severely complicates the derivation of self-consistent dynamical solution, but it is possible to extend the basic CAK formalism if one makes the simplifying assumption that the lines are uniformly distributed throughout the stellar spectrum (Friend \& Castor 1983). Using this approach, multi-line scattering models based on Monte Carlo simulation (Springmann 1994) and on non-isotropic diffusion analysis (Gayley et al. 1995) both show that effective trapping of radiation in the expanding envelope can lead to a more extended acceleration, with an effective velocity index up to $\beta \approx 2$ in the outer wind.

An overall point from such dynamical models based on line-driving is that it is quite difficult to obtain a velocity law with large velocity index, i.e. $\beta>2$. This is in part because line-driving itself depends on the velocity gradient, and so becomes inefficient in the extended, slow accelerations of a large $\beta$. Another fundamental factor regards the 
inverse-radius-squared fall-off of the radiative flux, $F \sim 1 / r^{2}$. Once the mass loss rate is tuned to allow the net outward force to be strong enough to overcome gravity near the stellar surface, the similar radial scaling of the radial flux and gravity tends then to make the net outward acceleration scale with the local inward acceleration of gravity. As shown above for the point-star CAK model, this leads to a velocity law with $\beta=1 / 2$, and the terminal speed scaling with the surface escape speed. Corrections due to finite-disk factor or multi-line scattering can extend the acceleration somewhat, but only up to velocity indices of order $\beta \approx 1-2$. Overall then, the very large velocity indices or associated acceleration scales inferred from apparent acceleration of subpeaks in WR $l p v$ are quite difficult to explain from dynamical models; this provides a key motivation for the present reexamination of these inferred extended acceleration scales.

\section{Line formation}

\subsection{Discrete LER without broadening or stellar occultation}

To provide a basis for the analysis of $l p v$, and its use as a diagnostic of flow acceleration, let us first examine how a mean, time-steady, line-profile depends on various wind properties. We again assume a spherically symmetric, monotonically expanding wind described by the standard $\beta$ velocity law, expressed in dimensionless form $w(r)=v(r) / v_{\infty}=\left(1-R_{*} / r\right)^{\beta}$ (Eq. (4)). As a simple first case, in this subsection we ignore the complications of stellar occultation and line broadening (i.e. by thermal or turbulent motions), and take the LER to have sharp boundaries defined by the lower and upper velocities $v_{\mathrm{l}}$ and $v_{\mathrm{u}}$. The subsequent subsections will examine the effects of modifying these simplifications.

Following Owocki \& Cohen (2001, Eq. (7)), and assuming optically-thin emission ${ }^{1}$, the total emitted luminosity at scaled wavelength $x=\left(\lambda / \lambda_{0}-1\right) c / v_{\infty}$ is given by,

$L_{\text {thin }}(x)=8 \pi^{2} C \int_{-1}^{1} \mathrm{~d} \mu \int_{r_{l}}^{r_{u}} \mathrm{~d} r r^{2} \rho^{2}(r) \delta(x+\mu w(r))$,

where $C$ is a constant that depends on the atomic process of emission (i.e. presently recombination). Performing the integral over $\mu$ gives

$L_{\text {thin }}(x) \propto \int_{r_{1}}^{r_{\mathrm{u}}} \mathrm{d} r H[w(r)-|x|] \frac{r^{2} \rho^{2}(r)}{w(r)}$,

where $H$ is the Heaviside function (unity for positive argument, zero otherwise). Note that from a shell at radius $r$, the contribution to the profile falls within the wavelength limits $\pm w(r)$.

Equation (16) applies in the case when both the line and continuum are optically thin. For the case of an optically thick line, the relative escape of photons along direction cosine $\mu$ is weighted by

$g(\mu, r)=\frac{1+\sigma(r) \mu^{2}}{1+\sigma(r) / 3}$

\footnotetext{
${ }^{1}$ Here and throughout our analysis, we ignore continuum absorption.
}

where $\sigma(r) \equiv \mathrm{d} \ln v / \mathrm{d} \ln r-1$. Integration over angle then gives (through the Dirac delta function) a corresponding weighting to the local emissivity, yielding for the optically thick emitted luminosity

$L_{\text {thick }}(x) \propto \int_{r_{1}}^{r_{\mathrm{u}}} \mathrm{d} r H[w(r)-|x|] \frac{r^{2} \rho^{2}(r)}{w(r)} g(|x| / w(r), r)$.

In the optically thin case (16), the wavelength $x$ enters only via the Heaviside function; thus the emission profile from each localized, narrow shell (extending from $r$ to $r+\mathrm{d} r$ ) is constant within the wavelength bounds $|x|<w(r)$ defined by the scaled velocity $w(r)$ of that shell. By contrast, in the optically thick case (18), there is an additional wavelength dependence via the relative escape function $g$; this gives a curvature to the shell emission profile, concave up in the inner wind where $\sigma(r)>0$, and concave down in the outer wind where $\sigma(r)<0$.

These properties of line formation are illustrated in Fig. 2. The bottom panels show the individual flux contributions when the LER is divided into seven discrete, finite shells spanning equal velocity bins. In the optically thin case (left column), the contributions are frequency independent, yielding flat-topped, box functions. Progressing from higher to lower velocity shells, these contributions narrow while their emission level increases, following the increased density in the slower, inner wind regions. The middle panels show a cumulative stack of these individual shell contributions, thus approximating the total emission profile of the upper panels, which are obtained by a full radial integration over the continuum of differential shells that span the LER. Note that the inner velocity of the LER sets the width of the flat top, while the outer velocity sets the maximum width of the profile down to the continuum level.

For an optically thick line (right column), the formation follows analogously, except that now the individual shell emission profiles can exhibit a net curvature within the box limits. In this particular example, wherein the LER spans the region where $\sigma(r)$ goes from positive to negative, the individual shell contributions go from concave up to concave down; the net cancellation of the two opposite senses of concavity gives the final profile a nearly flat-top form that is remarkably similar to the optically thin case. More generally, for optically thick lines formed predominantly in either the inner $(\sigma>0)$ or outer $(\sigma<0)$ regions, the total resulting profiles can exhibit either a double-peaked (concave up) form, or a rounded (concave down) form.

In the limiting case of a constant-speed outflow, we have $\sigma=-1$ and $g(\mu, r)=3\left(1-\mu^{2}\right) / 2$, yielding then upon angle integration a scaling $1-x^{2}$ for the $g$-factor in Eq. (18), which thus results in a parabolic shaped emission profile (see Ignace \& Gayley 2002).

Figure 3 compares optically thin line profiles computed for models with the same velocity range for the LER, but with much different velocity exponents, namely $\beta=1$ and $\beta=4$. Although these two values represent markedly different spatial variations of the velocity, with much slower overall acceleration for the $\beta=4$ case, the resulting line profiles are remarkably similar. This illustrates that the mean profile shape is quite insensitive to the wind acceleration, implying that inversion-based methods for inferring the wind velocity 

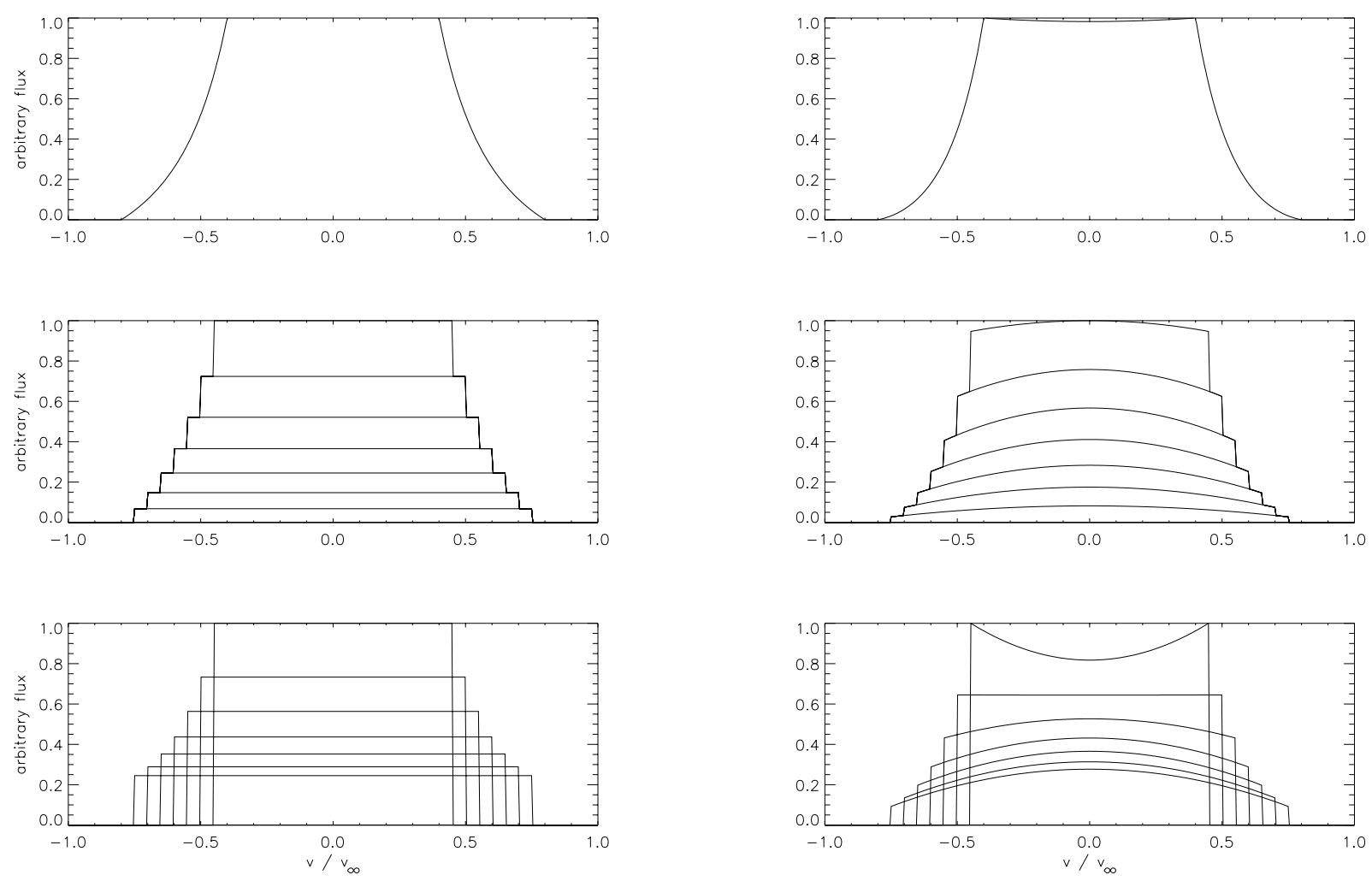

Fig. 2. For optically-thin (left) and optically-thick (right) lines, the bottom panels show the emission contribution from seven discrete subshells with equal velocity width spanning an LER between $40 \%$ and $80 \%$ of $v_{\infty}$ (with $\beta=1$ ). The middle panels then show a stacking of these subshell emissions to build a cumulative line profile that approximates the spatially integrated emission profile from the full LER, as given in the top panel.

law from the profile shape (Brown et al. 1997) are nearly degenerate with respect to the length scale of the velocity gain.

\subsection{Gaussian LER including stellar occultation}

Following NLTE model atmosphere results (Hillier 1988), as well as the convention from the previous $l p v$ analysis by LM, let us now specify the extent of the LER to follow a Gaussian form

$$
f(r)=\exp \left(-\left[\left(v(r)-v_{\mathrm{e}}\right) / \Delta v_{\mathrm{e}}\right]^{2}\right),
$$

where $\Delta v_{\mathrm{e}}$ is the velocity range for emission about the velocity centroid $v_{\mathrm{e}}$. For an outer wind velocity centroid $v_{\mathrm{e}}=0.9 v_{\infty}$ (and a relatively narrow velocity range of $\Delta v_{\mathrm{e}}=0.1 v_{\infty}$ ), Fig. 4 illustrates the resulting emission profiles for optically thin (dashed) and optically thick (solid) lines, comparing again a fast $(\beta=1$; upper panel) and a slow $(\beta=4$; lower panel) acceleration. The profiles are again all quite insensitive to the flow acceleration. Note that the Gaussian form of the LER now imparts a rounding to the edges of the flat portion for the thin lines, but for thick lines the already curved form of the profile makes this additional rounding less noticeable. The vertical dotted lines mark the velocity centroid. Note that in both cases this lies outside the width at half-maximum, which is commonly used in observational analyses as a rough measure of the LER velocity centroid; for thick lines, such use of half-maximum leads to a substantial underestimate of the characteristic velocity of the
LER. This can have important implications for the interpretation of $l p v$, as we discuss below (Sect. 4).

Figure 5 illustrates corresponding profiles for a line formed in the inner wind, with $v_{\mathrm{e}}=0.3 v_{\infty}$. For the optically thick lines, the double peaked profiles are the result of the concave-up form of the subshell emission, as discussed in Sect. 3.1; the marked Blue/Red asymmetry seen in the $\beta=1$ case arises from occultation of the redshifted emission from the hemisphere behind the star. Occultation also has a smaller, but still noticeable effect on the red edge of the optically thin line, giving the overall profile a modest blueward shift. By contrast, occultation effects are much reduced in the $\beta=4$ models, simply because the corresponding radius for the fixed velocity centroid $v_{\mathrm{e}}=0.3 v_{\infty}$ is now much further away from the star.

Such $\mathrm{B} / \mathrm{R}$ asymmetry is commonly observed in $\mathrm{O}$ supergiants, and so provides an useful constraint on the acceleration and velocity law (Puls et al. 1996; Crowther et al. 2002).

\subsection{Effect of emission profile broadening}

To account for the effects of finite broadening (by thermal or micro-turbulent motions), we next apply the ray-integration method of Castor (1970; based on the Sobolev approximation, Sobolev 1960), with the narrow (delta function) emission now replaced by a Gaussian of velocity width $v_{\text {turb }}$,

$\phi(x) \sim \exp \left[-\left(x v_{\infty} / v_{\text {turb }}\right)^{2}\right]$. 


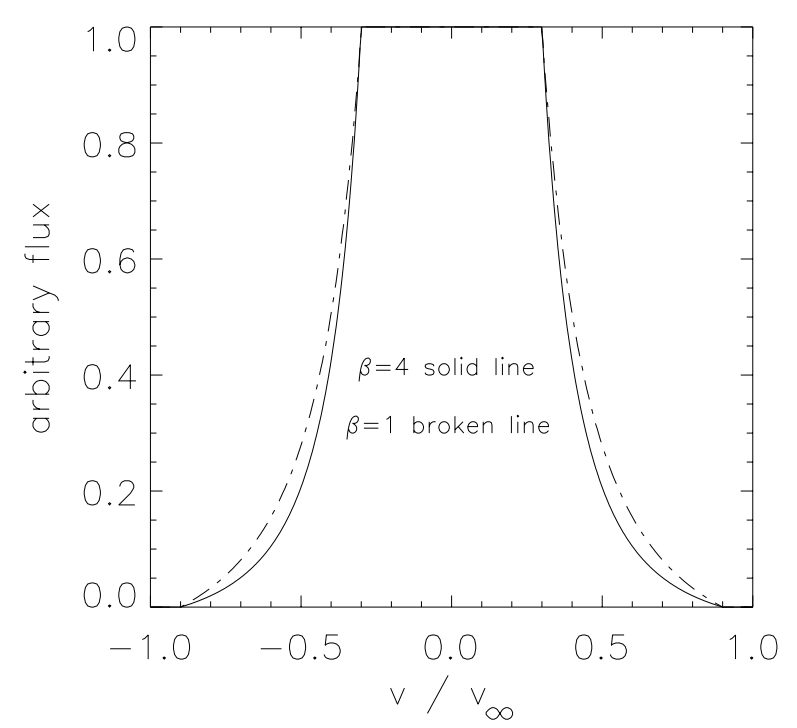

Fig. 3. Synthetic emission profiles for an optically-thin line forming between $30 \%$ and $90 \%$ of the terminal velocity but for two different velocity laws: the broken (solid) line corresponds to a steep (shallow) acceleration with $\beta=1(\beta=4)$. Apart from a modified maximum line-profile flux (not known a priori), the dependence of the line-wing shape on $\beta$ is weak, i.e. ca. $10 \%$ flux-difference for an extended LER (shown here) and decreasing for narrower LER. Inversion techniques (Brown et al. 1997) yield a very uncertain information on the wind velocity law.
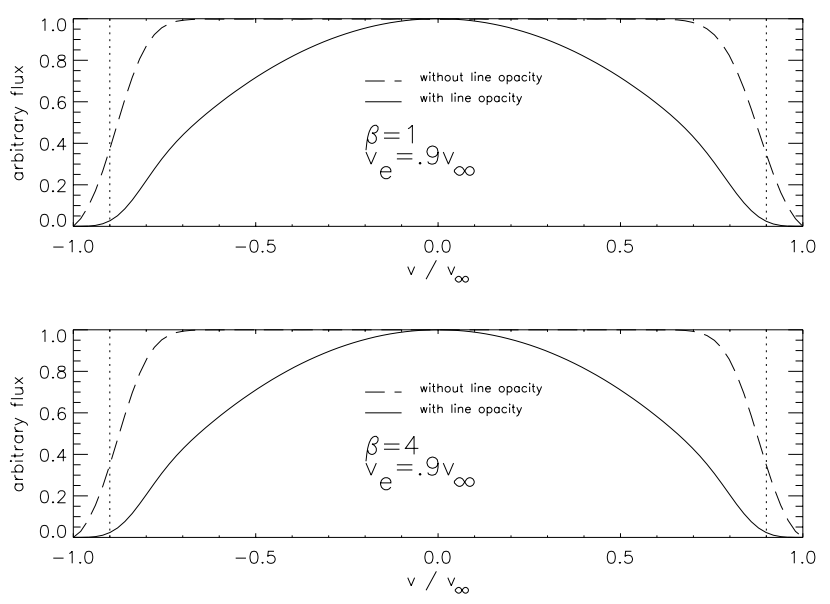

Fig. 4. Study of the impact of line optical depth effects and wind velocity law $(\beta=1,4)$ for a synthetic line whose LER is characterized by $v_{\mathrm{e}}=0.9 v_{\infty}$ and $\Delta v_{\mathrm{e}}=0.1 v_{\infty}$. Notice the rounding of the line-profile which shifts its HWHM velocity $0.3 v_{\infty}$ below the adopted $v_{\mathrm{e}}$. Note also the negligible impact of $\beta$ on the line-profile shape.

Figure 6 shows the optically thin emission profiles for models with a common velocity centroid $\left(v_{\mathrm{e}}=0.7 v_{\infty}\right)$, but with three distinct combinations of LER width and turbulent velocity. The solid and dashed curves compare a narrow $\left(\Delta v_{\mathrm{e}}=\right.$ $\left.0.1 v_{\infty}\right)$ and wide $\left(\Delta v_{\mathrm{e}}=0.3 v_{\infty}\right)$ LER, without any turbulence $\left(v_{\text {turb }}=0\right)$. Note that the latter gives a centrally peaked profile with very extended line wings; again the half-maximum width thus gives a severe underestimate of the LER velocity centroid. The dot-dashed curve shows the profile of a very narrow LER $\left(\Delta v_{\mathrm{e}}=0.05 v_{\infty}\right)$, but now with a substantial turbulent velocity
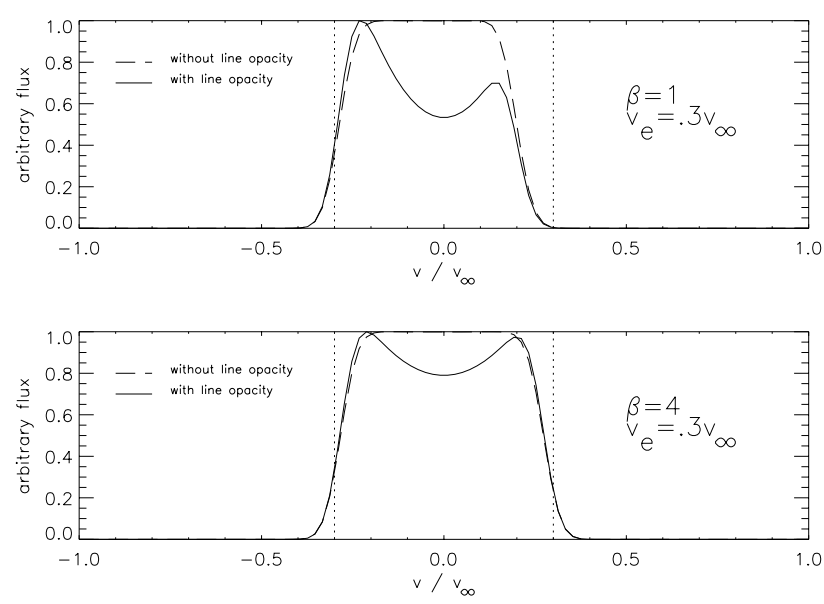

Fig. 5. Same as Fig. 4, but for an inner wind line with $v_{\mathrm{e}}=0.3 v_{\infty}$ and $\Delta v_{\mathrm{e}}=0.1 v_{\infty}$. The double-peaking discussed in Sect. 3.1 is strong, as well as a profile asymmetry resulting from disk-occultation. This latter effect, observed in $\mathrm{O}$ supergiants, would permit the constraint of the wind acceleration just above the hydrostatic photosphere.

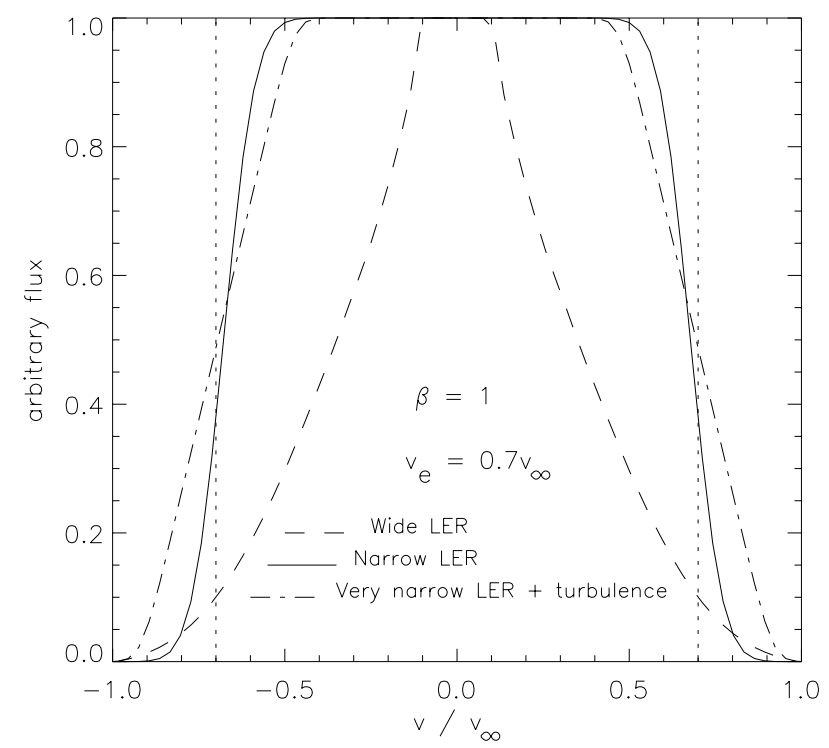

Fig. 6. Optically thin emission profiles for models with $\beta=1$ and velocity centroid $v_{\mathrm{e}}=0.7 v_{\infty}$, and three combinations of LER velocity width $\Delta v_{\mathrm{e}}$ and turbulent velocity $v_{\text {turb }}$. For cases without turbulence $\left(v_{\text {turb }}=0\right)$, the solid and dashed curves show results for a wide $\left(\Delta v_{\mathrm{e}}=\right.$ $\left.0.3 v_{\infty}\right)$ and narrow $\left(\Delta v_{\mathrm{e}}=0.1 v_{\infty}\right)$ LER. The dot-dashed curve is for a very narrow LER $\left(\Delta v_{\mathrm{e}}=0.05 v_{\infty}\right)$ but with substantial turbulence, $v_{\text {turb }}=0.2 v_{\infty}$. The vertical dotted line marks the velocity centroid $v_{\mathrm{e}}$. See Sect. 3.3 for further discussion.

$\left(v_{\text {turb }}=0.2 v_{\infty}\right)$; this turbulence further smoothes the edge of the profile, but does not substantially increase the overall profile width, even though such a turbulent velocity is at the upper limit of what is inferred in observational analyses (Robert 1992; LM). This latter point plays an important role in our revision of the LM analysis of $l p v$ (see Sect. 4).

We can conclude from this section that a detailed numerical approach is required for the determination of the LER characteristics of broad and rounded profiles, since these profiles may be subject to significant optical depth effects. Observed WR emission profiles showing a central peaking and broad 
wings reflect a line formation over an extended LER. Because they do not show any double-peaking, these lines are most likely optically-thin. The velocity at half-maximum is a poor indicator of the LER velocity centroid, due to the bias of profile flux in favor of inner wind regions. Broad flat top profiles are an unambiguous sign of optically thin line formation close to terminal velocity (otherwise they would be narrow with minute flat top portion) and over restricted ranges in velocity (otherwise they would be peaked). These latter signatures are the preferred diagnostics for $l p v$ studies.

\subsection{Application to line profiles observed at a time snapshot}

We are now in a position to use the model described above to assess the LER characteristics of time-snapshot observations of the recombination lines HeII5411 $\AA$ (WNs) and CIII5696 ^ (WCs) for seven WR stars: WR 103, WR 111 , WR 135, WR 136, WR 137, WR 138 and WR 140. This sample was chosen to match that used by Robert (1992) for her study of $l p v$ in WR stars, but the short-time exposures (ca. $30 \mathrm{~min}$.) we use here were obtained independently by P. Crowther (p.c.). (We exclude WR 40 from our sample because its HeII line shows a narrow P-Cygni profile for which our method does not apply.) Let us recapitulate the model assumptions. We adopt a spherically-symmetric monotonically-expanding outflow. Line emission occurs via recombination, thus possesses a density-square dependence (see Eq. (15)). No continuum opacity is included but allowance is made for variable line-opacity. Turbulence is treated as in Eq. (20). In Hillier (1988), a detailed description of the ionization stratification is made for WR stars in general, showing that it is indeed realistic to use a height segregation for recombination lines in WR atmospheres (see also Dessart et al. 2000). There is no explicit dependence of the emissivity on temperature, but it implicitly enters our treatment via this height segregation of wind ionization (Eq. (19)). We show our fits to single-exposure line profiles for these objects in Fig. 7, which shows the observed profiles (solid curves), together with our model fits (dot-dashed curves). (For clarity, we omit WR 135 from the figure; its profile resembles that of WR 111). The model parameters are presented in Table 1. The best fits are obtained by "eye-ball" estimate after iteration from a first guess, modifying the various parameters: line opacity, centroid and extent of the LER, without need to include any turbulence.

Note first that only for one case - WR 136 - is it necessary to invoke line optical depth effects; this contrasts with the claim that such effects can be responsible for outstanding fit discrepancies (LM). For candidates other than WR 136, we can identify some lines with extended line wings - for which we infer a broad LER (WR 103 and WR 138) - and others with near vertical line wings - for which we infer a narrow LER (the rest of the sample of stars); overall, the velocity width spans the range $\Delta v_{\mathrm{e}}=0.1-0.3 v_{\infty}$.

But a particularly important result is that the velocity centroids of the LERs are all substantially higher than inferred by $\mathrm{LM}$, in the range $v_{\mathrm{e}}=0.65-0.9 v_{\infty}$. Indeed, when these
Table 1. Parameters used in profile fitting. The modeled line is HeII5411 $\AA$ for WR 136 and WR 138, and CIII5696 ^ for all other cases. To substantiate the terminal velocities used in this study (first column), we compare values from LM (taken originally from from Prinja et al. (1990), labeled $a$ ), and from various other studies ( $b$ : Eennens \& Williams 1994; $c$ : Hillier \& Miller 1998; $d$ : Crowther $\&$ Smith 1996; $e$ : Howarth \& Schmutz 1992). Note that all values typically agree within ca. $100 \mathrm{~km} \mathrm{~s}^{-1}$. The last column denotes whether the fit requires an optically thick or thin line.

\begin{tabular}{|c|c|c|c|c|c|c|}
\hline WR & \multicolumn{3}{|c|}{$v_{\infty}$} & $v_{\mathrm{e}}$ & $\Delta v_{\mathrm{e}}$ & $\tau_{\text {line }}$ \\
\hline \multirow[t]{2}{*}{$\#$} & \multicolumn{3}{|c|}{$\mathrm{km} \mathrm{s}^{-1}$} & $v_{\infty}$ & $v_{\infty}$ & \\
\hline & This work & $\mathrm{LM}^{a}$ & diverse & & & \\
\hline 103 & 1200 & 1190 & $1100^{b}$ & .7 & .3 & thin \\
\hline 111 & 2400 & 2415 & $2300^{c}$ & .77 & .14 & thin \\
\hline 135 & 1525 & 1405 & $1525^{b}$ & .75 & .2 & thin \\
\hline 136 & 1850 & 1605 & $1750^{d}$ & .9 & .27 & thick \\
\hline 137 & 1900 & 1885 & $1900^{b}$ & .84 & .22 & thin \\
\hline 138 & 1400 & 1345 & $1400^{e}$ & .65 & .25 & thin \\
\hline 140 & 2900 & 2900 & $2870^{b}$ & .9 & .15 & thin \\
\hline
\end{tabular}

centroids are coupled with the inferred widths, i.e. $v_{\mathrm{e}}+\Delta v_{\mathrm{e}}$, we see that the line formation region in all cases extends essentially all the way to the terminal speed, $v_{\infty}$. This fact is manifest by the close correspondence between the maximum base width of these optical emission lines and the terminal speeds inferred by UV resonance lines.

Note that our detailed modeling is capable of fitting line profiles with a very high level of accuracy since minor changes to model parameters lead to very different synthetic line profiles; the fit quality is markedly degraded if either $v_{\mathrm{e}}$ or $\Delta v_{\mathrm{e}}$ are changed by even a few percents. The central part of the synthetic profiles for WR 103 and WR 138 show minute variations due to numerical inaccuracies, resulting from the strong emissivity gradient at the base of the LER, while that of WR 136 shows a slight Blue/Red asymmetry due to disk occultation effects (Sect. 3.2 and Fig. 5); In the present situations, such lowlevel irregularities, either real or from numerical error, would disappear with even a modest turbulence.

With such information on the velocity in the formation region of emission lines with observed $l p v$, we can now move on to using the wind acceleration derived from the migration of profile sub-peaks to infer the overall scale of the wind velocity law, as characterized by the value of $\beta R_{*}$.

\section{Application to inferred acceleration}

For these program stars, LM conducted an extensive analysis of the lpv observed by Robert (1992), inferring wind acceleration from the migration of profile sub-peaks, based on a phenomenological Discrete Wind Emission Element (DWEE) model. Using the inversion technique of Brown et al. (1997), LM estimated the LERs to extend over a narrow velocity range $\left(\Delta v_{\mathrm{e}} \approx 0.05 v_{\infty}\right)$ located relatively deep in the wind $\left(v_{\mathrm{e}}=\right.$ $0.4-0.8 v_{\infty}$ ), with turbulence claimed to be the main profile broadening mechanism. 

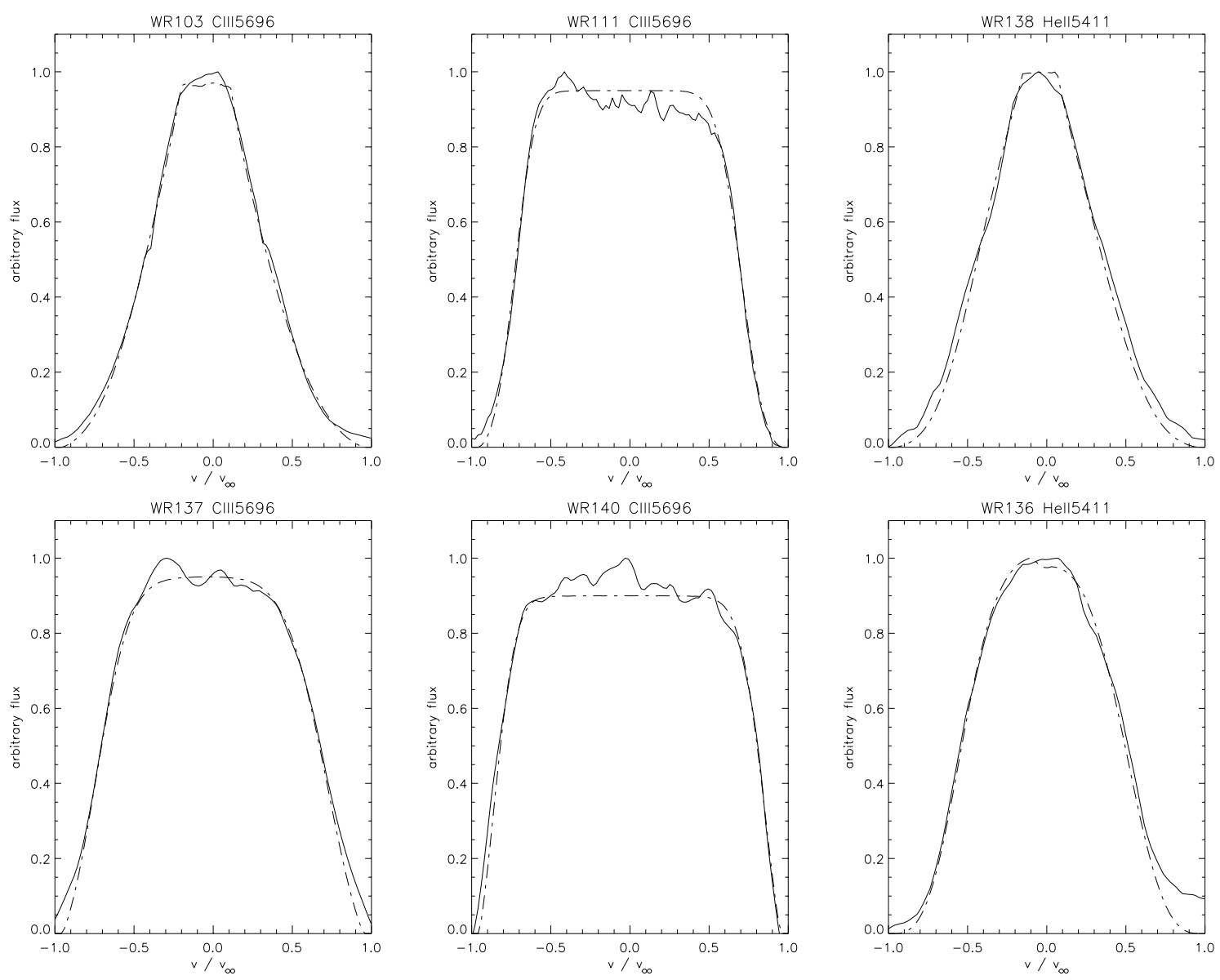

Fig. 7. Observed profiles (solid curves) and best fits (dot-dashed curves) in a chosen emission line (HeII 5411 Å or CIII $5696 \AA$ A) for six WR stars discussed in LM. The intensity has been rescaled so that the continuum is at zero and the maximum intensity is at unity. The wavelength is in velocity units, scaled to the terminal velocity of the corresponding line, as given in Table 1.

Such narrow LERs warranted taking a constant wind acceleration over its limited range, a key assumption for the degradation function method used to infer wind acceleration magnitudes. Combining these with the inferred LER velocities, LM concluded that the associated flow acceleration must extend over very large length scales, $\beta R_{*} \approx 20-80 R_{\odot}$. These are much larger than the estimated hydrostatic core radii of WR stars ( $<5 R_{\odot}$, Hillier \& Miller 1999; Dessart et al. 2000; Crowther et al. 2002), a result that challenges the standard radiative driving paradigm for these WR winds (Sects. 1-2).

By contrast, the LER characteristics derived here differ markedly from those inferred by LM, with systematically higher velocity centroids and larger widths, generally extending the outer range of the LER to nearly the terminal speed. Adopting the same acceleration magnitudes derived by LM, this now implies much more modest length scales for the wind acceleration. This follows simply from the fact that line formation in regions close to terminal speed can be expected to exhibit relatively low acceleration magnitudes.

These central results are illustrated in Fig. 8, wherein the left panel reproduces Fig. 14 of LM, while the right panel reconfigures the LER location and width according to the results given in our Table 1. Comparing the left to the right panel, note that the acceleration values are the same, but the LER velocity centroids (crosses) are shifted rightward (by ca. $0.1-0.3 v_{\infty}$ ) and the velocity ranges (horizontal bars) are substantially broadened (typically more than doubled). As in LM, the overplotted curves in both panels show the associated variation of acceleration vs. speed for models with various acceleration scales $\beta R_{*}$, labeled in units of $R_{\odot}$.

Note in particular that our LER parameters in the right panel are now consistent with substantially smaller acceleration lengths $\left(10-20 R_{\odot}\right)$ than implied by the LM values in the left panel $\left(20-60 R_{\odot}\right)$. These are still quite large, but perhaps now within reach of what radiative driving can achieve if one accounts for multi-line scattering (Springmann 1994; Gayley et al. 1995).

The origin of this difference lies solely in the new parameters derived for the LER velocity location and width. LM cited turbulence as the dominant source of broadening of WR line profiles; but it seems that with LM's adopted narrow and deep LER, line profiles with extended wings can only be fitted by invoking very large turbulent velocities, indeed much higher than those determined from their own $l p v$ analysis.

Figure 9 illustrates these points for the sample case of the HeII5411 A line from WR 138, comparing the observed profile (solid curves) with various models (dot-dashed curves). The top panels show our models with no turbulence (left) and 

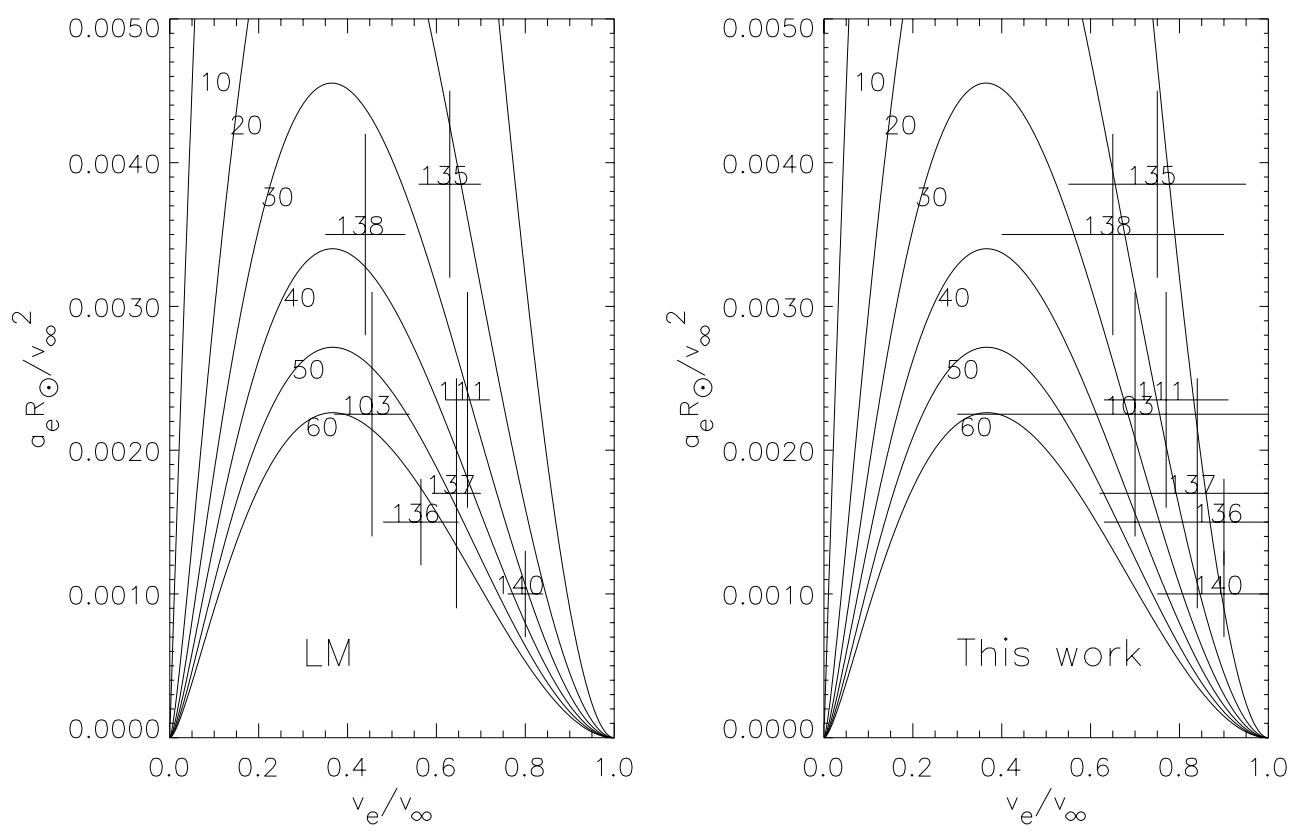

Fig. 8. Left: a reproduction of Fig. 14 of LM. This figure depicts the local wind acceleration $a_{\mathrm{e}}$ (determined from a degradation function analysis) at a given velocity $v_{\mathrm{e}}$ in the stellar atmosphere for seven stars analyzed in LM (crosses). The vertical error bar reflects an inaccuracy in the determination of the acceleration from the DWEE model of LM, while the horizontal error bar reflects the extent of, i.e. not an uncertainty in the position of, the LER in velocity space. The over-plotted curves are the theoretical $a_{\mathrm{e}}$ versus $v_{\mathrm{e}}$ for different values of $\beta R_{*}$. Right: Id. but with the LER characteristics determined in this work, i.e. crosses are shifted rightward by $.05-.25 v_{\infty}$ compared to LM, with considerably broader velocity ranges of the emission sites.

with the $l p v$-derived turbulence (right); both fits are quite good, though the turbulence introduces a smoothing and a moderately improved agreement. The bottom panels show LM models with this same lpv turbulence (left) and with the much larger turbulence needed to fit the observed profile (right); the former is the turbulence value cited by LM, but we clearly see that this does not provide an acceptable profile fit when combined with their inferred LER characteristics. Instead, a quite unrealistic turbulence is needed to fit the line.

In the present models with the line emission formed over a range of velocities, the emission profile is rounded even without turbulence (Fig. 9, panel A), and so the addition of a modest turbulence leads to only a modest additional broadening without much affecting the overall emission profile shape (cf. panels A and B of Fig. 9). By contrast, in the LM models, in which the LER is assumed to be at nearly a single velocity, the intrinsic profile has a more flat-topped, box shape, even with the modest level of turbulence (i.e. $v_{\text {turb }}=220 \mathrm{~km} \mathrm{~s}^{-1}$; Fig. 9 , panel C). Because of the relatively steep gradient in emission in going from the core to wing, the stronger level of turbulence $\left(v_{\text {turb }}=400 \mathrm{~km} \mathrm{~s}^{-1}\right)$ now has the general effect of redistributing flux from near the edges of the flat-top into the line wings; the overall effect then is to broaden the base (wing) of the profile, but round-off and narrow the emission core. An essential point is that the narrow range of velocity assumed by LM for the LER requires just such a large and physically dubious level of turbulence to produce the observed width and form of the line profile, whereas this can be produced naturally without invoking turbulence if one allows for the LER to span a modest range in outward flow speed.
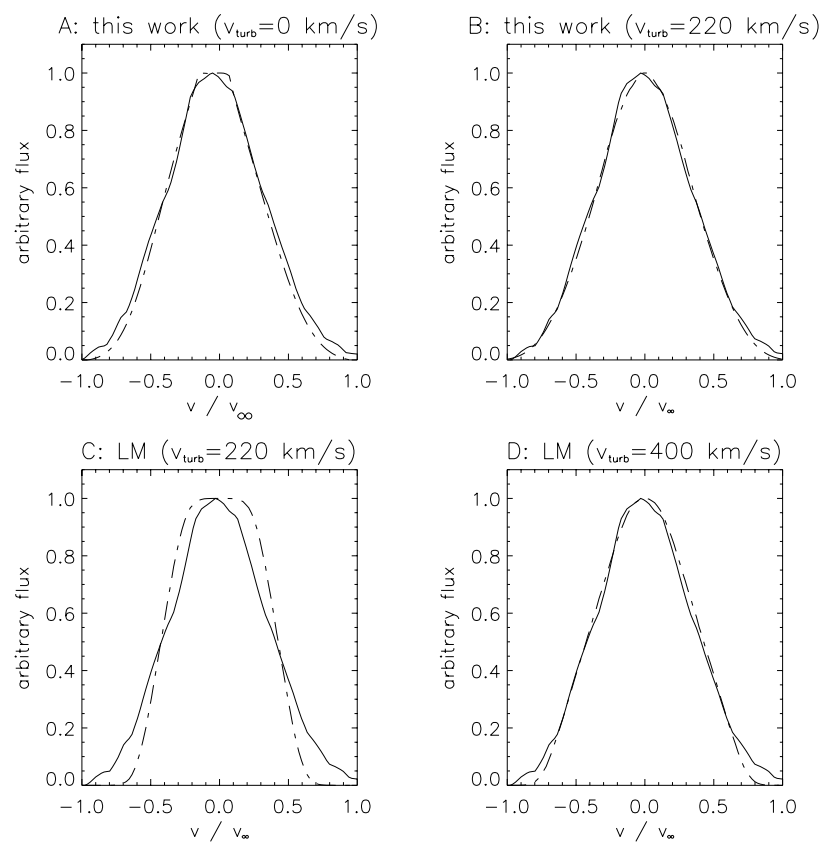

Fig. 9. Top: observed HeII5411 A profile of WR 138 (solid curves), compared with models using the parameters of the present study (dotdashed curves), without (A) and with (B, $v_{\text {turb }}=220 \mathrm{~km} \mathrm{~s}^{-1}$ ) turbulence. Bottom: same but with the parameters of LM (C), and with a turbulent velocity twice the value determined from the $l p v$ analysis $\left(\mathrm{D}, v_{\mathrm{turb}}=400 \mathrm{~km} \mathrm{~s}^{-1}\right)$.

This exercise supports the view (Hillier 1988) that WR line broadening results primarily from the finite extent of the LER in velocity space, and not from turbulent broadening. These changes relative to the LER properties used by LM may not 

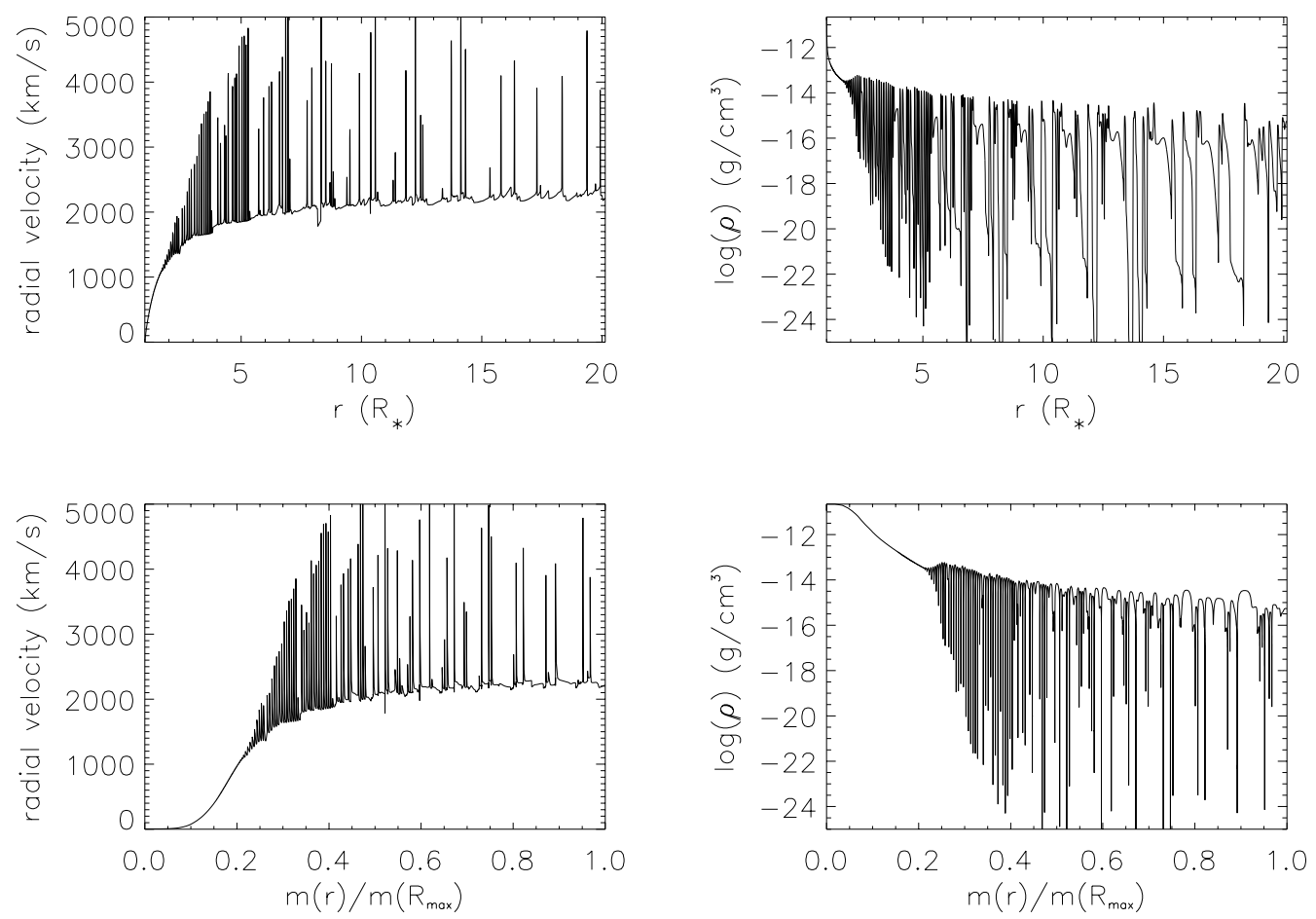

Fig. 10. Single time-snapshot from the hydrodynamics simulation of the radiative instability, showing the velocity (left column) and log-density (right column), in Eulerian (top) and Lagrangian (bottom) coordinates.

have much effect on the acceleration magnitudes derived in their $l p v$ analysis (see Sect. 5), but they do imply a substantial downward revision (by a factor of two or more) in their quoted acceleration scales, i.e. from $20-80 R_{\odot}$ to $10-20 R_{\odot}$.

\section{Wind acceleration inferred from synthetic Ipv}

The migrating sub-peaks observed in $l p v$ are understood to stem from the variable emissivity of the structured wind accelerating through the LER (Sect. 1). Lucy (1984) and Owocki \& Rybicki (1984) proposed that this wind structure could result from the strong instability to small-scale perturbations of the line-driving mechanism. The non-linear evolution of a line-driven wind subject to such an instability was studied for the first time in a $1 \mathrm{D}$ radiation-hydrodynamics computation by Owocki et al. (1988), showing that the outflow relaxes into a highly-developed non-monotonic radial velocity field, whose associated shocks lead to radially-confined slow high density compressions separated by extended fast rarefied regions. We present these properties for one such simulation of an O-star wind (with suitable parameters for the supergiant star $\zeta$ Puppis) in the top panels of Fig. 10 for the radial velocity (left) and density (right) as a function of radius. When plotted in a Lagrangian coordinate (bottom panels, Dessart \& Owocki 2002b), the bulk of the material follows a velocity, which, when time-averaged, matches to within a few percents the CAK velocity law with a $\beta$-exponent value of 0.8 . In other words, only a negligible fraction of the total wind mass departs from such a CAK velocity, contributing relatively little flux to (density-squared dependent) emission profiles. Thus, this property justifies the use of a standard $\beta$-velocity law for the wind structures, as done in LM for the modeling of $l p v$.
However, because the lines used by LM have a finite rather than a narrow LER (Sect. 4), assuming a fixed wind acceleration may lead to systematic errors in the derived acceleration magnitudes. Indeed, in the case, e.g., of a wide LER, most of the profile flux originates from the low-velocity high-density regions (Sect. 3), which for a fully clumped wind, tends to favor the variability signal of those deep wind regions. In this section, to circumvent such a kind of biases, we present a more direct comparison between observed and synthetic $l p v$. To do so, we compute the optically-thin line emissivity arising from a 3D wind assumed to be composed of independent star-centered cones characterized by a fixed angular extent. The crux of this so-called "Patch" method (described in great details in Dessart \& Owocki 2002a, hereafter DO) is to adopt, for each cone, a purely $1 \mathrm{D}$ wind layout as computed by radiation hydrodynamics simulations of the radiative instability. A representative snapshot of the height variation of the velocity and density is shown in Fig. 10, both in Eulerian and Lagrangian coordinates. Thus, while the method retains some 1D features as far as the gas is concerned, it allows us to introduce a complete wind anisotropy, which, as illustrated in DO, is required by the lack of emission profile variations in the line center region. Moreover, Dessart \& Owocki (2002b) interpreted the velocity-scale of variable profile sub-peaks at line-center as arising from the lateral geometrical broadening of wind structures. They showed that with a patch-size of $3 \mathrm{deg}$, one obtains a satisfactory fit to the observed sub-peak velocity width at line center as well as the fluctuation magnitude of observed $l p v$. Thus, the same value of $3 \mathrm{deg}$ is used here.

In the following two sections, we use the Patch method together with the hydrodynamical simulations of the radiative 

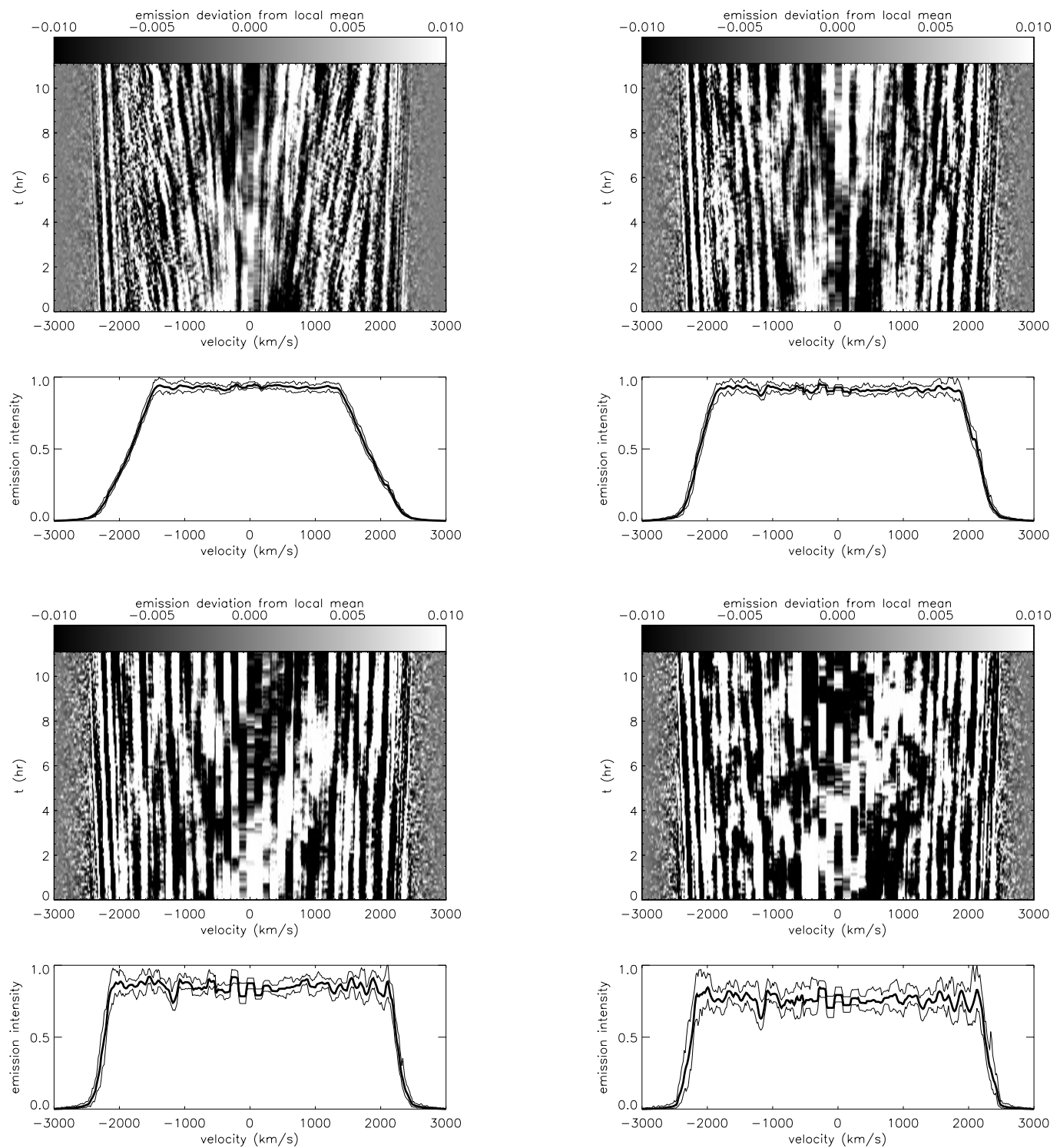

Fig. 11. Montage of synthetic lpv based on radiation-hydrodynamics simulations of the line-driving instability for four optically thin emission lines whose LER extend out to $20 R_{*}$ but start at different depths: 2.5 and $5.0 R_{*}$ (top left and right panels) and 10.0 and $15.0 R_{*}$ (bottom left and right panels). To enhance the visibility of migrating sub-peaks in the grayscales, the local maximum flux deviation is limited to $1 \%$.

instability presented in Fig. 10 to compute synthetic lpv of WR and $\mathrm{O}$ stars. Strictly speaking, such hydrodynamical simulations only apply to winds driven via single line-scattering, i.e. O star winds, and not to WR winds whose larger mass loss rates require the treatment of multiple line-scattering (Gayley et al. 1995). Nonetheless, both are understood to be line-driven and believed to share similar dynamical properties, differences arising from the height location of line-emission: all O-star optical diagnostics originate close to the hydrostatic core radius (below ca. 2-3 $R_{*}$, Hillier et al. 2002) while those of WRs subsist out to large radii. It is via such a height segregation that we now study such a synthetic $l p v$ for WR and O star emission lines.

\subsection{Measured sub-peak acceleration from synthetic Ipv: WR-star case}

We stressed in the previous sections the importance of using a reliable LER characterization for the interpretation of $l p v$. In particular, we found that in general, the lines studied by LM formed over a finite LER that extended out to terminal velocity. Hence, here, for all profile computations, we set the outer LER radius at the maximum radius of the hydrodynamical simulation of $20 R_{*}$, corresponding to the terminal velocity of the flow within a few percents. We then vary the inner radius of the LER to reproduce the range of observed widths of $l p v$ diagnostics, covering the values $r_{1}=2.5,5,10$ and $15 R_{*}$. We show a sequence of synthetic grayscale time-series in Fig. 11, with increasing $r_{1}$ value from top to bottom, and left to right.

In the top-left panel, we see the presence of sub-peaks with a range of acceleration magnitudes. Around line of sight velocities of $1000 \mathrm{~km} \mathrm{~s}^{-1}$ and $-1000 \mathrm{~km} \mathrm{~s}^{-1}$, we can estimate values of ca. $50 \mathrm{~km} \mathrm{~s}^{-1} / \mathrm{h}$, which due to a line of sight angle cosine weight of about a half, correspond to an higher intrinsic wind acceleration of ca. $30 \mathrm{~m} \mathrm{~s}^{-2}$. In the line wing, the sub-peak migration magnitude is systematically lower, ca. $20 \mathrm{~km} \mathrm{~s}^{-1} / \mathrm{h}$ and 

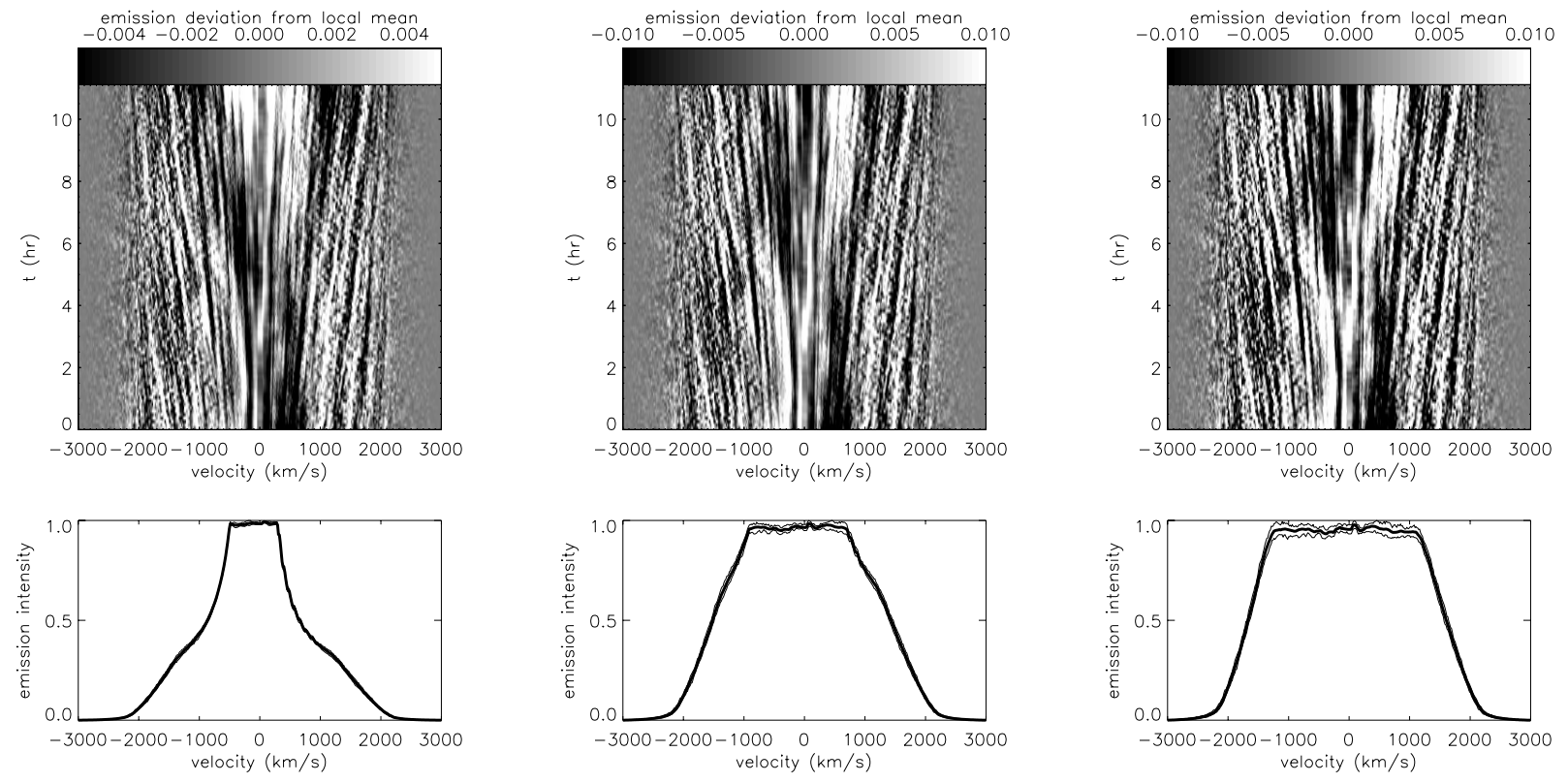

Fig. 12. Same as previous figure but for 3 emission line profiles whose LER starts at 1.2, 1.5 and $2.0 R_{*}$ (left to right) but extends out to the same outer radius, i.e. $10.0 R_{*}$.

since these profile patterns arise from wind emitting structures advecting directly towards us, the sub-peak acceleration corresponds directly to the intrinsic wind acceleration, i.e. of ca. $8 \mathrm{~m} \mathrm{~s}^{-2}$. This is consistent with the fact that the LER is wide, covering a large range of intrinsic wind accelerations. Despite additional geometrical effects, this leads to a decreasing subpeak migration magnitude from line center to line wing.

As we move the inner edge of the LER to larger radii (subsequent three panels), the fast migration of sub-peaks in the flat part of the profile is at first less conspicuous (top-right panel), before disappearing altogether for outer wind forming lines (bottom panels). In these latter cases, the LER covers a narrow range of velocities and is well characterized by a unique wind acceleration directly measurable from line-wing subpeaks. Contrary to the case of a deep forming line, the profile migration now increases from line center to line wing. Its magnitude is small, consistent with the fact that the LER now probes wind regions that are within $10 \%$ of the terminal velocity.

Interestingly, the simulation corresponding to a line whose LER starts at $2.5 R_{*}$ (top-left panel) reproduces well both the temporal evolution as well as the time-averaged line emission observed for CIII5696 $\AA$ for WR 111 . In particular, the migration magnitude of ca. $30 \mathrm{~m} \mathrm{~s}^{-2}$ is in good agreement with values given in LM as well as the observed sub-peak migration in the flat part of the profile. Note that the migrating subpeaks appearing in the line wing are unobserved, a likely consequence of the fact that $1 \mathrm{D}$ radiation hydrodynamics simulations underestimate the magnitude of radial velocity dispersion. But the more dramatic drawback from our model is that we use a stellar radius of $19 R_{*}$, while the hydrostatic core radius of WR 111 is expected to be a factor of 2-10 smaller (Hillier \& Miller 1999). Hence, with such small core radii, the inferred WR wind acceleration length scale is still significantly more extended than expected from radiation driven wind theory which at present favors a "standard" velocity law with $\beta$ of ca. 1-2 at most.

\subsection{Measured sub-peak acceleration from synthetic Ipv: O-star case}

In the previous section, we chose an inner radius for the LER compatible with the generally large emission line profile widths of WR stars. By contrast, in O-stars, emission line formation starts right at the base of the outflow, resulting in much narrower emission profiles, usually quite pointed rather than flat-topped. Thus because the whole wind is optically thin, a given emission line carries information on all wind heights, allowing us to constrain the inner wind acceleration from single-exposure line profiles (Sect. 3.2; Puls et al. 1996; Crowther et al. 2002), as well as from lpv (Eversberg et al. 1998).

To model the $l p v$ of O-star winds, we use the same approach as in Sect. 5.1, this time setting the inner edge of the LER to very small wind heights, covering the values $1.2,1.5$ and $2.0 R_{*}$ (left to right panels in Fig. 11). The migration magnitude of profile sub-peaks is quite comparable between all three simulations, and only slightly higher that for the situation where the LER starts at $2.5 R_{*}$ (Sect. 5.1). In our model (Fig. 10), although the wind acceleration magnitude increases towards the wind base, the wind ceases to be clumped below ca. $2 R_{*}$. Hence, no profile variations arise from wind emission at these depths, invalidating the use of our method based on $l p v$ to infer the wind acceleration.

But, although the $l p v$ pattern is very similar in these three cases, the time-averaged line profile changes dramatically as the LER inner radius decreases. Indeed, the line-wing shape presents a very clear kink, at a profile location which corresponds to the wind velocity at which clumping appears. The 

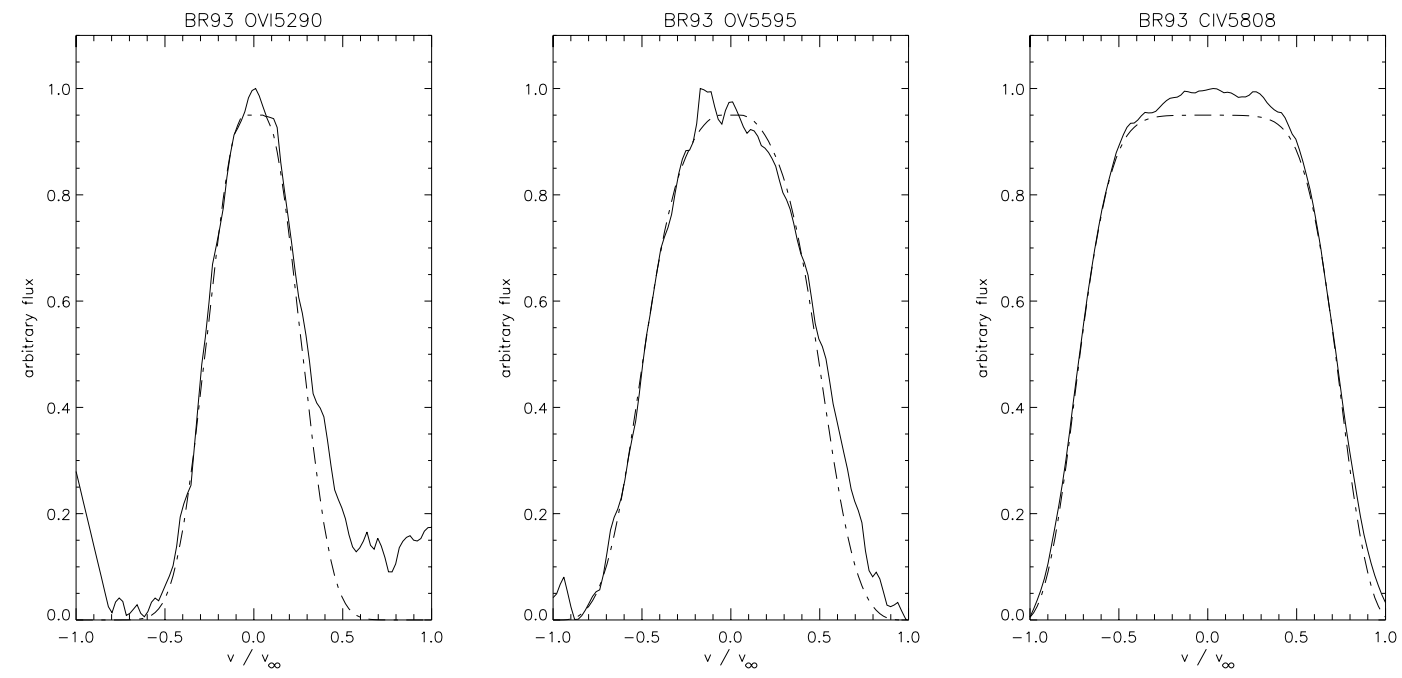

Fig. 13. Optical emission lines of Ovi5290 A, Ov5595 $\AA$ and CIv5808 $\AA$ observed in the Br93 spectrum. Superposed on each profile is a satisfactory fit with the model described in Sect. 3.3. The velocity centroid $v_{\mathrm{e}}$ of the LER for the three modeled lines, going from left to right, are $40 \%, 65 \%$ and $85 \%$ of $v_{\infty}$, while the velocity range of the LER is $20 \%$ of $v_{\infty}$ for each. No line opacity needed to be accounted for in order to reproduce the profile. Some turbulence is included: $v_{\text {turb }} \sim 400 \mathrm{~km} \mathrm{~s}^{-1}$, which corresponds to $10 \%$ of the terminal velocity. This illustrates why WO stars represent ideal candidates to reveal differential wind acceleration from lpv.

emissivity of the $l p v$ diagnostics being density-squared dependent, it is suddenly enhanced when going from the unclumped inner wind regions through the clumped outer wind ones. Thus, if O-star wind clumping only started at a sizeable fraction of the terminal velocity, this feature would be observable.

However, to our knowledge, such a profile kink has not been seen in O-star spectra which might be supporting the idea that their winds are clumped even in the inner wind region. The observed $l p v$ for the $\mathrm{O}$ supergiant star $\zeta$ Puppis (Eversberg et al. 1998) also suggested that the pattern of migrating subpeaks was compatible with a wind clumping starting right at the wind base. Moreover, Prinja et al. (2001) reported a B/R asymmetry in the $l p v$ of HD 152408 (O8:Iafpe or WN9ha), with the migration magnitude of profile sub-peaks twice as small in the red part than in the blue part of the line profile. In Sect. 3.2, we showed that disk occultation can lead to a significant flux deficit in the red part of the profile. But if this deficit is combined to a $\mathrm{B} / \mathrm{R}$ dichotomy in $l p v$, it also implies that the occulted innermost wind regions are also clumped. Thus, these various elements support the spectroscopic analysis of $\mathrm{O}$ supergiant stars (Hillier et al. 2002, 2003; Crowther et al. 2002) which require that the wind clumping starts right from the base.

\section{Summary and future work}

We have reexamined the LER characteristics of WR lpv diagnostics (Robert 1992). We find that, in general, these lines are optically thin, showing a profile broadening that results from the finite extent of the LER rather than from turbulence. Furthermore, we find that they form much closer to terminal velocity than proposed in previous studies (LM), leading to a downward revision of the WR wind acceleration length scales $\beta R_{*}$ from $20-80 R_{\odot}$ to $10-20 R_{\odot}$. Based on $1 \mathrm{D}$ radiation hydrodynamics simulations of the radiative instability and the "Patch" method, we compute synthetic lpv for CIII5696 ^ for WR 111. We are able to reproduce well the observed $l p v$ for that star, although our model uses an O-star core radius. Both evolutionary and spectroscopic models predict small WR core radii, a few times the solar value, which thus makes WR wind acceleration length scales still considerably extended, although not to the extent proposed by LM. Forthcoming VLTI-AMBER observations will hopefully give an accurate evaluation of the spatial scales of WR stars and their associated winds, thereby setting the magnitude of the discrepancy between the inferred WR wind acceleration from observations and that expected from radiation driven wind theory.

In our synthetic $l p v$ simulations for optically thin O-star winds, we find a clear signature for the onset velocity of wind clumping, which results from the strong sensitivity of recombination line emissivity to variations in wind density. This introduces a kink in the line-wing profile shape, making the clumped outer wind regions relatively more emissive than the smooth inner wind ones. The absence of such a kink in even high-resolution spectroscopic observations of $\mathrm{O}$ supergiants together with results from $l p v$ studies of such stars (Eversberg et al. 1998; Prinja et al. 2001) suggest that indeed, wind clumping may well start right from the hydrostatic base in $\mathrm{O}$ supergiant stars.

Finally, the WR-lpv dataset obtained so far uses diagnostic lines that form close to terminal velocity; such lines contain only poor information on the overall wind acceleration. Using optically thinner winds would yield a more valuable information on the wind acceleration. This is the case for OB objects but their wind density is however so low that no line emission arises from above ca. 2-3 $R_{*}$ (Hillier et al. 2002), providing no information on the outer wind dynamics. On the contrary, WO star winds have not only a low optical depth in the visual band but they also show strong optical emission lines of markedly different velocity widths, resulting from a strong wind ionization stratification (Crowther et al. 2000). Indeed, using the model of Sect. 3.3, we find that optical 
emission lines of oxygen and carbon form in regions located between $40 \%$ to $100 \%$ of the terminal velocity (Fig. 13). Thus, WO stars constitute excellent targets for $l p v$ studies to provide further observational constraints on the structure and dynamics of WR star winds.

Acknowledgements. S.P.O. acknowledges support of NASA grant NAGW5-3530 and NSF grant AST-0097983, awarded to the University of Delaware.

\section{References}

Brown, J. C., Richardson, L. L., Cassinelli, J. P., \& Ignace, R. 1997, A\&A, 325, 677

Castor, J. I., Abbott, D. C., \& Klein, R. I. 1975, ApJ, 195, 157 (CAK)

Crowther, P. A., \& Smith, L. J. 1996, A\&A, 305, 541

Crowther, P. A., Fullerton, A. W., Hillier, D. J., et al. 2000, ApJ, 538, L51

Crowther, P. A, Hillier, D. J., Evans, C. J., et al. 2002, ApJ, 579, 774

Dessart, L. 1999, Ph.D. Thesis, Univ. London

Dessart, L., \& Owocki, S. P. 2002a, A\&A, 383, 1113 (DO)

Dessart, L., \& Owocki, S. P. 2002b, A\&A, 393, 991

Dessart, L., \& Owocki, S. P. 2003, A\&A, 406, 1

Eversberg, T., Lépine, S., \& Moffat, A. F. J. 1998, ApJ, 494, 799

Eenens, P. R. J., \& Williams, P. M. 1994, MNRAS, 269, 1082
Friend, D. B., \& Abbott, D. C. 1986, ApJ, 311, 701

Friend, D. B., \& Castor, J. I. 1983, ApJ, 272, 259

Gayley, K. G., Owocki, S. P., \& Cranmer, S. R. 1995, ApJ, 442, 296 Hillier, D. J. 1988, ApJ, 327, 822

Hillier, D. J., \& Miller, D. L. 1998, ApJ, 496, 407

Hillier, D. J., \& Miller, D. L. 1999, ApJ, 519, 354

Hillier, D. J., Lanz, T., Heap, S. R., et al. 2003, ApJ, 588, 1039

Howarth, I. D., \& Schmutz, W. 1992, A\&A, 261, 503

Ignace, R., \& Gayley, K. G. 2002, ApJ, 568, 954

Langer, N., Hamann, W.-R., Lennon, M., et al. 1994, A\&A, 290, 819

Lépine, S. 1998, Ph.D. Thesis, Université de Montréal

Lépine, S., \& Moffat, A. F. J. 1999, ApJ, 514, 909 (LM)

Lucy, L. B. 1984, ApJ, 274, 372

Owocki, S. P., \& Rybicki, G. B. 1984, ApJ, 284, 337

Owocki, S. P., Castor, J. I., \& Rybicki, G. B. 1988, ApJ, 335, 914

Owocki, S. P., \& Cohen, D. H. 2001, ApJ, 558, 802

Pauldrach, A. W. A., Puls, J., \& Kudritzki, R. P. 1986, A\&A, 164, 86

Prinja, R. K., Barlow, M. J., \& Howarth, I. D. 1990, ApJ, 361, 607

Prinja, R. K., Stahl, O., Kaufer, A., et al. 2001, A\&A, 367, 891

Puls, J., \& Kudritzki, R. 2000, ARA\&A, 38, 613

Puls, J., Kudritzki, R.-P., Herrero, A., et al. 1996, A\&A, 367, 171

Robert, C. 1992, Ph.D. Thesis, Université de Montréal

Schaerer, D., \& Maeder, A. 1992, A\&A, 263, 129

Sobolev, V. 1960, Moving Envelopes of Stars (Cambridge, Mass.: Harvard Univ. Press)

Springmann, U. 1994, A\&A, 289, 505 